\title{
Comparación entre técnicas de modelado computacional de edificios de tipo muro
}

Comparison between techniques of computational modeling of wall-type buildings

\section{Sergio Álvarez González}

Estudiante de Ingeniería Civil

Universidad de Costa Rica, Costa Rica

sergiogabriel.alvarez@ucr.ac.cr

Fecha de recepción: 31 octubre 2018 / Fecha de aprobación: 8 marzo 2019

Índices y Bases de Datos:

\section{latindex ucrindex \\ - Dialnet DOA}

(c) revistas.ucr.ac.cr/index.php/materiales

(c) lanamme.ucr.ac.cr

@ metodosymateriales.lanamme@ucr.ac.cr
Políticas de Uso:

\section{(c) $\$$}

Revista Métodos y Materiales por LanammeUCR se distribuye bajo: Licencia Creative Commons Atribución-NoComercialSinDerivar 4.0 Internacional. ISSN electrónico: 2215-4558 


\title{
Comparación entre técnicas de modelado computacional de edificios de tipo muro
}

\section{Comparison between techniques of computational modeling of wall-type buildings}

\author{
Sergio Álvarez González \\ Estudiante de Ingeniería Civil \\ Universidad de Costa Rica, Costa Rica \\ sergiogabriel.alvarez@ucr.ac.cr
}

Fecha de recepción: 31 octubre 2018 / Fecha de aprobación: 8 marzo 2019

\section{RESUMEN}

En este artículo se compara la aplicación de diferentes técnicas de modelado computacional de edificios de tipo muro; específicamente se estudiaron las siguientes metodologías de modelado:

a) modelado a través de elementos tipo barra con sección transversal rectangular

b) modelado a través de elementos tipo barra con sección transversal irregular

c) modelado a través de elementos tipo área

Con el fin de aplicar cada una de las técnicas de modelado a distintos edificios se utilizó el software de análisis estructural llamado ETABS. En el cual se efectuó un análisis dinámico espectral de cada uno de estos modelos, con lo cual se creó una matriz de resultados que permitió comparar y discutir las diferentes respuestas estructurales globales que se pueden obtener para un mismo edificio cuando se realiza un análisis lineal utilizando distintas técnicas de modelado. De esta comparación se concluye que en general los modelos compuestos por elementos tipo barra con sección transversal rectangular y los modelos elaborados con elementos tipo área presentan comportamientos similares, por lo que se podrían modelar edificios de tipo muro de hasta 30 niveles con ambas metodologías sin esperar diferencias significativas en las respuestas estructurales globales comparadas (períodos, fuerzas de sismo en la base de la estructura y derivas). Por otro lado, los modelos elaborados con elementos tipo barra con sección transversal irregular presentaron diferencias significativas para estas mismas respuestas.

PALABRAS CLAVE: Modelaje de muros; análisis de edificios; técnicas de modelado; ingeniería estructural.

\section{ABSTRACT}

This paper compares the application of different computational modeling techniques of wall-type buildings; specifically, the following modeling methodologies were studied:

a) modeling through frame type elements with rectangular cross-section

b) modeling through frame type elements withirregular cross-section

c) modeling through shell type elements

In order to apply each modeling techniques to different buildings, the structural analysis software called ETABS was used. In which a spectral dynamic analysis of each one of these models was carried out, with which a results matrix was created that allowed comparing and discussing the different global structural responses that can be obtained for the same building when a linear analysis is carried out using different modeling techniques. From this comparison, it is concluded that in general, the models composed of frame type elements with a rectangular crosssection and the models made with area type elements show similar behaviors, so that wall-type buildings of up to 30 levels could be modeled with both methodologies without significant differences in structural responses (periods, seismic forces at the base of the structure and drifts). On the other hand, models made with frame type elements with irregular cross-section presented significant differences for these same responses.

KEYWORDS: Wall modeling; building analysis; modeling techniques; structural engineering. 


\section{INTRODUCCIÓN}

Durante la etapa de diseño y análisis de todo tipo de edificaciones es común emplear programas computacionales que permitan determinar parámetros referentes al comportamiento de las estructuras ante las posibles cargas a las cuales estarán sometidas. Gran parte de estos programas especializados en ingeniería estructural y sísmica utilizan el método del elemento finito (o MEF por sus siglas en español) para el análisis de dichas estructuras. Este es un método numérico de uso frecuente para la solución de problemas ingenieriles de gran complejidad tanto en el rango lineal como en el no-lineal (Chandrupatla \& Belegundu, 2011) y el cual permite conocer los desplazamientos y esfuerzos internos en elementos sólidos, lineales (entre ellos elementos tipo barra) y de tipo área o "cascarón", como lo son los muros y losas de entrepiso.

La teoría desarrollada para elementos tipo barra se ajusta adecuadamente al comportamiento exhibido por elementos esbeltos como las vigas y columnas, donde el efecto de la flexión interna rige sobre los efectos producidos por las fuerzas cortantes. En contraparte la teoría utilizada para el análisis de elementos tipo área es aplicable al caso de elementos chatos como los muros, donde las deformaciones por esfuerzos cortantes son significativas (Chocano, 2003; Popov, 2000). Aun así, es posible modelar muros mediante ambos conceptos de modelado, esto trae consigo variaciones en cuanto a los modos de oscilación, períodos, fuerzas sísmicas y derivas de una edificación que pueden incidir posteriormente en la etapa de diseño (Tan \& Kubin, 2008; Ammar\& Hassan, 2011). Aunque en la actualidad el uso de elementos tipo barra para el modelado de muros en edificaciones es cada vez menos frecuente, esta sigue conformando una técnica que en ocasiones puede llegar a ser empleada en oficinas de diseño debido su menor costo computacional, lo que motiva la realización de esta investigación.

Por lo tanto, a raíz de las diferencias que pueden existir en la respuesta estructural de un edificio de tipo muro dado el uso de diversos conceptos de modelado, surge la necesidad de conocer las implicaciones de la utilización de las técnicas de modelado más habituales en los resultados que se obtienen a partir de un análisis de elemento finito efectuado en un programa computacional especializado. Para ello se utilizó el software de análisis estructural llamado ETABS con el fin de efectuar el modelado de distintas edificaciones (ficticias y reales) a través de diferentes técnicas. Posteriormente realizando un análisis dinámico espectral de cada uno de estos modelos se creó una matriz de resultados que permitió comparar y discutir las diferentes respuestas estructurales globales que se pueden obtener para un mismo edificio cuando se utilizan distintas técnicas de modelado.

\section{EDIFICACIONES MODELADAS}

Para esta investigación se modelaron un total de doce edificaciones, de las cuales las seis primeras corresponden a edificios ficticios con distribuciones en planta simplesylas seis restantes a edificios reales con distribuciones de muros mucho más complejas. Se consideraron estructuras que van desde los 5 hasta los 30 niveles y con alturas de piso de $3 \mathrm{~m}$. La cantidad de niveles utilizado para cada edificación se muestra en el Tabla 1, mientras que la Figura 1 muestra las distribuciones en planta de muros para cada estructura.

\section{Tabla 1. Cantidad de niveles por edificio}

\begin{tabular}{|c|c|}
\hline Modelo & Cantidad de niveles \\
\hline Edificio $A$ & 5 \\
\hline Edificio $B$ & 10 \\
\hline Edificio $C$ & 15 \\
\hline Edificio D & 20 \\
\hline Edificio $E$ & 25 \\
\hline Edificio $F$ & 30 \\
\hline Edificio $G$ & 5 \\
\hline Edificio $H$ & 10 \\
\hline Edificio $~$ & 15 \\
\hline Edificio J & 20 \\
\hline Edificio $K$ & 25 \\
\hline Edificio $L$ & 30 \\
\hline
\end{tabular}




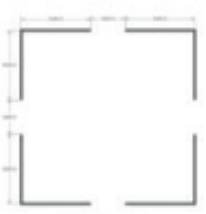

Edificio A

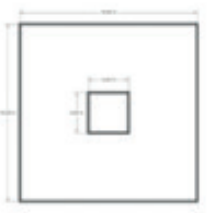

Edificio B

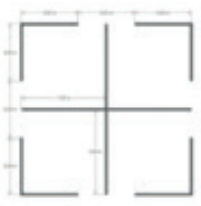

Edificio C

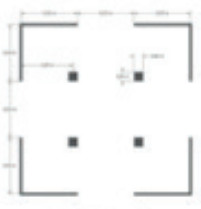

Edificio D

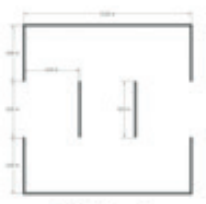

Edificio $E$

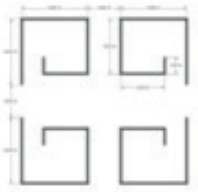

Edificio $F$

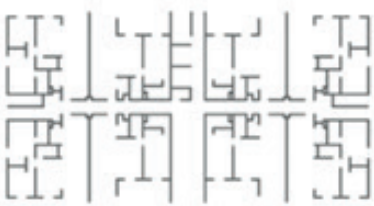

Edificio G

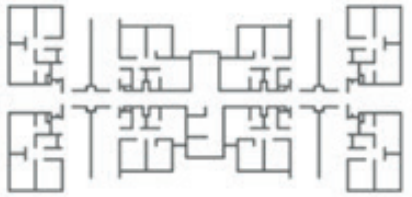

Edificio $\mathrm{H}$

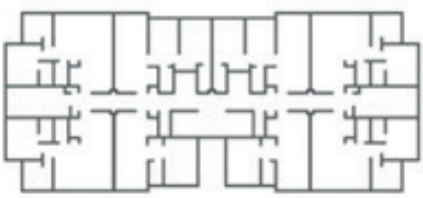

Edificio I

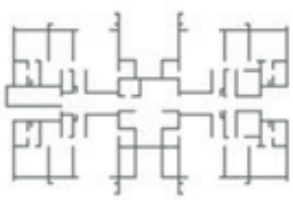

Edificio J

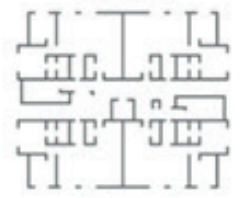

Edificio $\mathrm{K}$

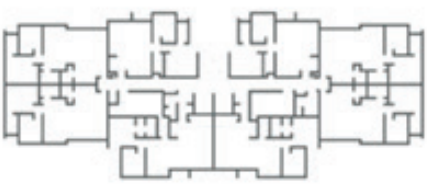

Edificio L

Figura 1. Distribuciones de muros en planta de edificaciones ficticias y reales

\section{CONSIDERACIONES REALIZADAS}

Dentro de las consideraciones generales realizadas en el modelado de las estructuras se tiene:

\subsection{Materiales}

El único material utilizado en el modelado fue el concreto reforzado; cuyas propiedades se muestran en el Tabla 2.

\begin{tabular}{|c|c|}
\hline \multicolumn{2}{|c|}{ Tabla 2. Propiedades del concreto } \\
\hline Densidad & $2400 \mathrm{~kg} / \mathrm{m}^{3}$ \\
\hline Resistencia (f'c) & $21 \mathrm{MPa}$ \\
\hline Módulo de elasticidad & $21459 \mathrm{MPa}$ \\
\hline Módulo de Poisson & 0,2 \\
\hline Módulo de cortante & $8941 \mathrm{MPa}$ \\
\hline
\end{tabular}




\subsection{Agrietamiento}

Se consideraron secciones de muro sin agrietar. Esta consideración, aunque contradice lo indicado en el CSCR2010 (CFIA, 2014) no influye en las conclusiones presentadas en esta investigación, debido a que el objetivo es realizar una comparación relativa entre las respuestas estructurales globales que se pueden obtener para una misma edificación por el uso de diferentes técnicas de modelado.

\subsection{Cargas permanentes y temporales}

Las cargas del peso propio de las estructuras se calcularon automáticamente por el programa computacional ETABS (CSi, 2015); considerando espesores de $200 \mathrm{~mm}$ para muros y entrepisos. El resto de cargas asignadas corresponden a cargas típicas de edificaciones cuyo uso es de apartamentos. Las cargas permanentes de elementos no estructurales pueden observarse en el Tabla 3, mientras que la carga temporal se estableció con una magnitud de $200 \mathrm{~kg} / \mathrm{m}^{2}$ (Tabla 6.1 del CSCR-2010). Dichas cargas se asignaron como cargas uniformemente distribuidas sobre los entrepisos.

\begin{tabular}{|c|c|}
\hline \multicolumn{2}{|c|}{$\begin{array}{c}\text { Tabla3. Cargas permanentes de elementos no } \\
\text { estructurales }\end{array}$} \\
\hline Cargas permanentes por metro cuadrado & Peso $\left(\mathrm{kg} / \mathrm{m}^{2}\right)$ \\
\hline Peso de los cerramientos livianos & 50 \\
\hline Peso de los sistemas electromecánicos & 15 \\
\hline Peso de acabados de piso de concreto & 75 \\
\hline Nivelación de piso de concreto $(3 \mathrm{~cm})$ & 70 \\
\hline Cielorraso de láminas de yeso ("gypsum”) & 15 \\
\hline Total & 225 \\
\hline
\end{tabular}

\subsection{Zona sísmica y sitio de cimentación}

Debido a que la zona sísmica y sitio de cimentación no son variables relevantes dentro de la investigación, se estableció que todas las estructuras se encuentranen la Zona III y en un sitio de cimentación tipo $\mathrm{S}_{3}$. Realizar esta simplificación permitió trabajar todos los modelos en un mismo contexto, lo cual representó una ventaja debido a que fija estas variables para efectos comparativos. Según la Tabla 2.3 del CSCR2010, la aceleración pico efectiva de diseño para un período de retorno de 475 años y las condiciones de sitios definidas, corresponde a un 36\% de la aceleración de la gravedad $\left(9,81 \mathrm{~m} / \mathrm{s}^{2}\right)$; así mismo los factores espectrales dinámicos (FED) referentes a las condiciones del sitio establecidas se obtuvieron de la Figura 5.7 del CSCR-2010, donde se utilizó una ductilidad de "1", por lo cual las fuerzas obtenidas no corresponden a fuerzas de diseño.

\subsection{Coeficiente sísmico}

No se tomaron en cuenta aspectos de sobrerresistencia en la estructura, por lo que el denominador de la ecuación para el cálculo del coeficiente sísmico se tomó igual a la unidad. Nuevamente, aunque dicha consideración no es acorde con lo indicado en el CSCR-2010, esta no influye en la comparación relativa de los resultados obtenidos entre las diferentes técnicas de modelado. Por otro lado, el factor de importancia se definió con una magnitud de " 1 ", el cual corresponde a edificaciones de ocupación normal según la tabla 4.1 del CSCR-2010.

$$
\mathrm{C}=\frac{\mathrm{a}_{\mathrm{ef}} \cdot \mathrm{FED} \cdot \mathrm{I}}{\mathrm{SR}}
$$

Dónde:

$\mathrm{a}_{\mathrm{ef}}=$ aceleración pico efectiva de diseño.

$\mathrm{I}=$ factor de importancia.

FED = factor espectral dinámico.

$\mathrm{SR}=$ factor de sobrerresistencia.

Adicionalmente las demandas sísmicas horizontales se tomaron como la suma vectorial de los efectos en una dirección más el 30\% de este efecto en la otra dirección.

\subsection{Masa del edificio considerada en el análisis por sismo}

Para el cálculo de la carga sísmica se consideró 100\% de las cargas permanentes y $15 \%$ de las cargas temporales (6.1.3 de CSCR-2010).

\subsection{Modos de oscilación}

La cantidad de modos asignados en los modelos se hizo de manera que se obtuviera una masa efectiva oscilante acumulada del $90 \%$ de la masa total en cada dirección ortogonal.

\subsection{Tipos de elementos}

Los entrepisos se modelaron como elementos de tipo cascarón delgado, los cuales funcionan como losa en dos direcciones, además estos se consideraron como diafragmas rígidos en su plano. Por otro lado, dependiendo de la técnica utilizada los 
muros se modelaron con elementos de tipo barra o elementos de tipo área, en cuyo caso se definieron como elementos de tipo cascarón delgado. Finalmente, los apoyos se consideraron como empotramiento perfecto.

\subsection{Discretizado de elementos}

Todos los elementos de tipo área utilizados en muros y entrepisos se discretizaron en elementos de 1,00 m x 1,00 m. Tales dimensiones se definieron con base en un análisis de sensibilidad efectuado para dicha variable, del cual se encontró que elementos de menor tamaño presentaban diferencias poco significativas en los resultados (menores al 5\%) y en contraparte incrementaban sustancialmente el costo computacional.

\section{TÉCNICAS ESTUDIADAS}

Cada una de las distribuciones de muros mostradas anteriormente en la Figura 1, se modelaron en el programa computacional ETABS utilizando las tres diferentes técnicas descritas a continuación:

\subsection{Modelado con elementos tipo barra de sección transversal rectangular}

Se definieron los diferentes muros como elementos tipo barra con sección transversal rectangular; estos se vincularon entre sí a través de elementos tipo barra conocidos como "brazos rígidos" (ver Figura 2), los cuales cumplen la función de vigas "infinitamente" rígidas que permiten modelar una unión apropiada entre muro-muro y muro-entrepiso. Para estos elementos en particular se introdujo un modificador de la inercia a flexión dentro de sus propiedades; dicho valor se tomó como la razón de inercias de las áreas transversales del muro con mayor inercia dentro de la planta de muros del modelo y la inercia de la sección transversal del elemento tipo barra definido como "brazo rígido" multiplicada por un factor de 10 000. El brazo rígido únicamente emula la unión que existe entre los distintos componentes de la estructura, por lo que se modeló como un elemento sin masa ni peso. Finalmente se asignó un valor de 10000 al resto de modificadores de sus propiedades.

\subsection{Modelado con elementos tipo barra de sección transversalirregular}

Para este caso se definieron elementos tipo barra con secciones transversales que siguieran la forma de todo un conjunto de muros adyacente entre sí; por lo tanto, para este escenario se definieron elementos con secciones transversales en forma de "L" "T" o incluso geometrías más irregulares como en el caso de los edificios reales. El procedimiento general seguido para vincular los diferentes elementos dentro del modelo fue el mismo que cuando se utilizaron elementos tipo barra con secciones transversales de forma rectangular.

\subsection{Modelado con elementos tipo área}

Cómo se indicó anteriormente, se definieron los muros como elementos tipo área (cascarón delgado) y se discretizaron en elementos de tamaños de 1,00 m x 1,00 m.

La Figura 2 muestra una vista de los modelos del Edificio A ejecutados a través de las diferentes técnicas. Como puede observarse para el caso del modelo ejecutado través de elementos tipo barra de sección trasversal rectangular se utilizan dos elementos para conformar la sección de muros en forma "L", mientras que en el modelado a través de elementos tipo barra con sección transversal irregular se utiliza un único elemento cuya sección transversal sea en forma de "L".

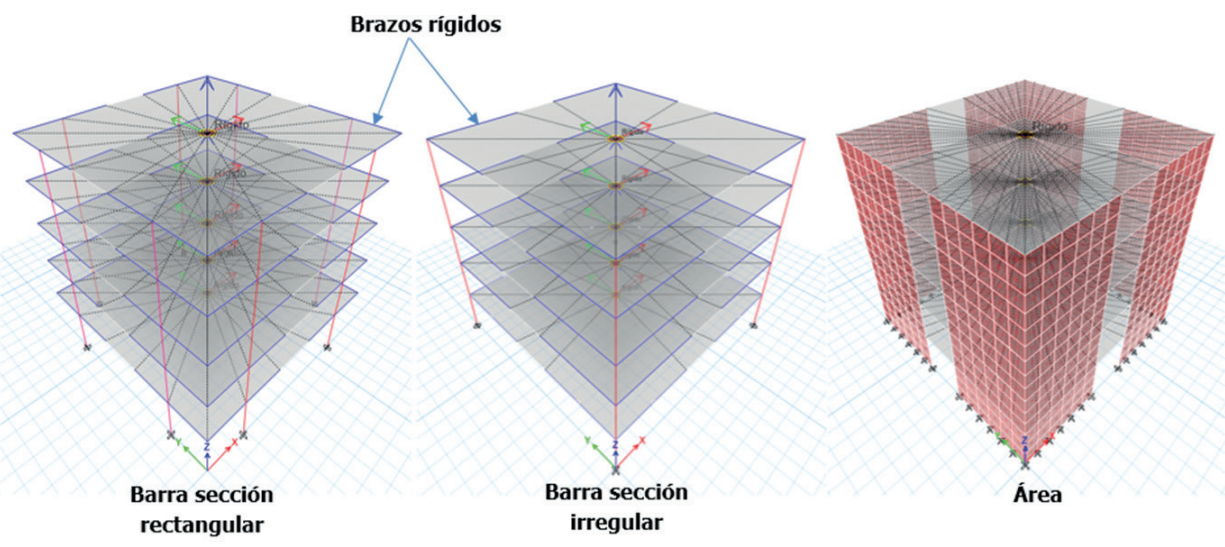

Figura 2. Vistas generales de las técnicas de modelado utilizadas (Edificio A) 


\section{RESULTADOS}

En las Tablas 4 y 5 se resumen las respuestas estructurales globales obtenidas (períodos, fuerzas de sismo en la base de la estructura y derivas) para los edificios ficticios y reales a través de las diferentes técnicas de modelado estudiadas. Es importante mencionar que para efectos comparativos se tomó como referencia los modelos de elementos de tipo área, calculando la diferencia porcentual entre modelos de la siguiente manera:

$$
\% \frac{\mathrm{X}_{\text {Módelo Área }}-\mathrm{X}_{\text {Módelo Barra }}}{\mathrm{X}_{\text {Módelo Área }}} \cdot 100 \%
$$

Dónde:

$\mathrm{X}_{\text {Módelo Área' }} \mathrm{X}_{\text {Módelo Barra }}$ Representa cualquier parámetro de respuesta estructural obtenido con la técnica respectiva.

\begin{tabular}{|c|c|c|c|c|c|c|c|}
\hline \multicolumn{8}{|c|}{ Valor del periodo principal de oscilación (s) } \\
\hline \multirow[t]{2}{*}{ Edificio } & \multirow{2}{*}{$\begin{array}{l}\text { Cantidad } \\
\text { de niveles }\end{array}$} & \multicolumn{2}{|c|}{$\begin{array}{l}\text { Modelo: } \\
\text { Área } \\
\end{array}$} & \multicolumn{2}{|c|}{$\begin{array}{l}\text { \%Diferencia Modelo: } \\
\text { Barra rectangular }\end{array}$} & \multicolumn{2}{|c|}{$\begin{array}{l}\text { \%Diferencia Modelo: } \\
\text { Barra irregular }\end{array}$} \\
\hline & & $\mathrm{X}$ & $\mathbf{Y}$ & $\mathrm{X}$ & $\mathrm{Y}$ & $\mathrm{X}$ & $\mathrm{Y}$ \\
\hline A & 5 & 0,170 & 0,170 & $1,76 \%$ & $1,76 \%$ & $-4,12 \%$ & $-4,12 \%$ \\
\hline B & 10 & 0,221 & 0,221 & $6,33 \%$ & $6,33 \%$ & $-4,52 \%$ & $-4,52 \%$ \\
\hline $\mathrm{C}$ & 15 & 0,695 & 0,695 & $2,16 \%$ & $2,16 \%$ & $-4,75 \%$ & $-4,75 \%$ \\
\hline D & 20 & 1,933 & 1,933 & $1,29 \%$ & $1,29 \%$ & $-9,98 \%$ & $-9,98 \%$ \\
\hline $\mathbf{E}$ & 25 & 2,094 & 1,142 & $2,39 \%$ & $2,80 \%$ & $-8,21 \%$ & $-9,62 \%$ \\
\hline $\mathbf{F}$ & 30 & 2,363 & 1,995 & $0,89 \%$ & $1,55 \%$ & $-20,31 \%$ & $-25,41 \%$ \\
\hline \multicolumn{8}{|c|}{ Cortantes basales en la estructura $(\mathrm{kN})$} \\
\hline \multirow[t]{2}{*}{ Edificio } & \multirow{2}{*}{$\begin{array}{l}\text { Cantidad } \\
\text { de niveles }\end{array}$} & \multicolumn{2}{|c|}{$\begin{array}{l}\text { Modelo: } \\
\text { Área }\end{array}$} & \multicolumn{2}{|c|}{$\begin{array}{l}\text { \%Diferencia Modelo: } \\
\text { Barra rectangular }\end{array}$} & \multicolumn{2}{|c|}{$\begin{array}{l}\text { \%Diferencia Modelo: } \\
\text { Barra irregular }\end{array}$} \\
\hline & & $\mathrm{Vx}$ & $V_{y}$ & $\mathrm{Vx}$ & $V_{y}$ & $\mathrm{Vx}$ & $V_{y}$ \\
\hline A & 5 & 7564 & 7564 & $0,77 \%$ & $0,77 \%$ & $0,40 \%$ & $0,40 \%$ \\
\hline B & 10 & 17766 & 17766 & $0,82 \%$ & $0,82 \%$ & $-1,71 \%$ & $-1,71 \%$ \\
\hline $\mathrm{C}$ & 15 & 21847 & 21847 & $-1,10 \%$ & $-1,10 \%$ & $6,50 \%$ & $6,50 \%$ \\
\hline D & 20 & 11968 & 11968 & $-0,42 \%$ & $-0,41 \%$ & $6,16 \%$ & $6,16 \%$ \\
\hline $\mathbf{E}$ & 25 & 22622 & 14450 & $-1,41 \%$ & $-0,71 \%$ & $5,08 \%$ & $10,79 \%$ \\
\hline $\mathrm{F}$ & 30 & 21435 & 19669 & $-0.09 \%$ & $-0.01 \%$ & $18.00 \%$ & $9.50 \%$ \\
\hline \multicolumn{8}{|c|}{ Momentos basales en la estructura ( $\mathrm{kN}-\mathrm{m})$} \\
\hline \multirow{2}{*}{ Edificio } & \multirow{2}{*}{$\begin{array}{l}\text { Cantidad } \\
\text { de niveles }\end{array}$} & \multicolumn{2}{|c|}{$\begin{array}{l}\text { Modelo: } \\
\text { Area }\end{array}$} & \multicolumn{2}{|c|}{$\begin{array}{l}\text { \%Diferencia Modelo: } \\
\text { Barra rectangular }\end{array}$} & \multicolumn{2}{|c|}{$\begin{array}{l}\text { \%Diferencia Modelo: } \\
\text { Barra irregular }\end{array}$} \\
\hline & & $\mathrm{Mx}$ & My & $M x$ & My & $\mathrm{Mx}$ & My \\
\hline A & 5 & 84150 & 84150 & $0,67 \%$ & $0,67 \%$ & $0,47 \%$ & $0,47 \%$ \\
\hline $\mathbf{B}$ & 10 & 371083 & 371083 & $0,50 \%$ & $0,50 \%$ & $-1,17 \%$ & $-1,17 \%$ \\
\hline $\mathrm{C}$ & 15 & 677420 & 677420 & $-1,63 \%$ & $-1,63 \%$ & $6,61 \%$ & $6,61 \%$ \\
\hline D & 20 & 389310 & 389310 & $-1,36 \%$ & $-1,36 \%$ & $12,56 \%$ & $12,56 \%$ \\
\hline$E$ & 25 & 585520 & 1040341 & $-1,91 \%$ & $-2,46 \%$ & $9,21 \%$ & $8,25 \%$ \\
\hline $\mathrm{F}$ & 30 & 869902 & 1017199 & $-0,84 \%$ & $-1,20 \%$ & $25,73 \%$ & $34,52 \%$ \\
\hline \multicolumn{8}{|c|}{ Derivas } \\
\hline \multirow[t]{2}{*}{ Edificio } & \multirow{2}{*}{$\begin{array}{l}\text { Cantidad } \\
\text { de niveles }\end{array}$} & \multicolumn{2}{|c|}{$\begin{array}{l}\text { Modelo: } \\
\text { Area }\end{array}$} & \multicolumn{2}{|c|}{$\begin{array}{l}\text { \%Diferencia Modelo: } \\
\text { Barra rectangular }\end{array}$} & \multicolumn{2}{|c|}{$\begin{array}{l}\text { \%Diferencia Modelo: } \\
\text { Barra irregular }\end{array}$} \\
\hline & & $\mathrm{X}$ & $\mathbf{Y}$ & $\mathrm{x}$ & $\mathrm{Y}$ & $\mathrm{X}$ & $\mathrm{Y}$ \\
\hline A & 5 & 0,000682 & 0,000682 & $0,29 \%$ & $0,29 \%$ & $-9,38 \%$ & $-9,38 \%$ \\
\hline B & 10 & 0,000505 & 0,000505 & $2,18 \%$ & $2,18 \%$ & $-13,66 \%$ & $-13,66 \%$ \\
\hline $\mathrm{C}$ & 15 & 0,003670 & 0,003670 & $-1,09 \%$ & $-1,09 \%$ & $-13,65 \%$ & $-13,65 \%$ \\
\hline D & 20 & 0,008009 & 0,008009 & $-1,74 \%$ & $-1,74 \%$ & $-17,37 \%$ & $-17,37 \%$ \\
\hline$E$ & 25 & 0,003996 & 0,006098 & $1,48 \%$ & $2,18 \%$ & $-8,00 \%$ & $-14,58 \%$ \\
\hline$F$ & 30 & 0,005272 & 0,006566 & $1,31 \%$ & $0,21 \%$ & $-39,64 \%$ & $-33,83 \%$ \\
\hline
\end{tabular}




\begin{tabular}{|c|c|c|c|c|c|c|c|}
\hline \multicolumn{8}{|c|}{ Valor del periodo principal de oscilación (s) } \\
\hline \multirow[t]{2}{*}{ Edificio } & \multirow{2}{*}{$\begin{array}{l}\text { Cantidad } \\
\text { de niveles }\end{array}$} & \multicolumn{2}{|c|}{$\begin{array}{l}\text { Modelo: } \\
\text { Área }\end{array}$} & \multicolumn{2}{|c|}{$\begin{array}{l}\text { \%Diferencia Modelo: } \\
\text { Barra rectangular }\end{array}$} & \multicolumn{2}{|c|}{$\begin{array}{l}\text { \%Diferencia Modelo: } \\
\text { Barra irregular }\end{array}$} \\
\hline & & $\mathrm{X}$ & $\mathbf{Y}$ & $\mathrm{x}$ & $\mathbf{Y}$ & $\mathrm{X}$ & $\mathrm{Y}$ \\
\hline G & 5 & 0,148 & 0,112 & $6,08 \%$ & $5,36 \%$ & $-12,84 \%$ & $-14,29 \%$ \\
\hline $\mathbf{H}$ & 10 & 0,260 & 0,308 & $4,23 \%$ & $0,00 \%$ & $-13,85 \%$ & $-19,16 \%$ \\
\hline I & 15 & 0,306 & 0,466 & $6,54 \%$ & $-1,07 \%$ & $-16,01 \%$ & $-19,53 \%$ \\
\hline $\mathrm{J}$ & 20 & 0,605 & 0,759 & $5,29 \%$ & $2,11 \%$ & $-9,09 \%$ & $-16,73 \%$ \\
\hline $\mathbf{K}$ & 25 & 1,088 & 1,299 & $2,39 \%$ & $0,15 \%$ & $-19,21 \%$ & $-22,48 \%$ \\
\hline $\mathbf{L}$ & 30 & 0,88 & 1,574 & $3,07 \%$ & $0,00 \%$ & $-8,64 \%$ & $-18,81 \%$ \\
\hline \multicolumn{8}{|c|}{ Cortantes basales en la estructura $(\mathrm{kN})$} \\
\hline \multirow[t]{2}{*}{ Edificio } & \multirow{2}{*}{$\begin{array}{l}\text { Cantidad } \\
\text { de niveles }\end{array}$} & \multicolumn{2}{|c|}{$\begin{array}{l}\text { Modelo: } \\
\text { Area }\end{array}$} & \multicolumn{2}{|c|}{$\begin{array}{l}\text { \%Diferencia Modelo: } \\
\text { Barra rectangular }\end{array}$} & \multicolumn{2}{|c|}{$\begin{array}{l}\text { \%Diferencia Modelo: } \\
\text { Barra irregular }\end{array}$} \\
\hline & & $\mathbf{V x}$ & $\mathrm{V}_{\mathbf{y}}$ & $\mathrm{Vx}$ & Vy & $\mathrm{Vx}$ & Vy \\
\hline $\mathbf{G}$ & 5 & 39642 & 36827 & $4,51 \%$ & $6,59 \%$ & $-0,59 \%$ & $-6,87 \%$ \\
\hline $\mathbf{H}$ & 10 & 78225 & 74811 & $3,49 \%$ & $1,88 \%$ & $-3,32 \%$ & $1,87 \%$ \\
\hline 1 & 15 & 89762 & 85425 & $3,98 \%$ & $0,86 \%$ & $-1,96 \%$ & $0,06 \%$ \\
\hline $\mathrm{J}$ & 20 & 128805 & 100062 & $-4,16 \%$ & $-2,52 \%$ & $-0,62 \%$ & $13,55 \%$ \\
\hline $\mathbf{K}$ & 25 & 56221 & 48412 & $-0,54 \%$ & $0,50 \%$ & $21,89 \%$ & $22,63 \%$ \\
\hline L & 30 & 134436 & 90487 & $0.42 \%$ & $1.81 \%$ & $11.96 \%$ & $13.53 \%$ \\
\hline \multicolumn{8}{|c|}{ Momentos basales en la estructura (kN-m) } \\
\hline \multirow[t]{2}{*}{ Edificio } & \multirow{2}{*}{$\begin{array}{c}\text { Cantidad } \\
\text { de niveles }\end{array}$} & \multicolumn{2}{|c|}{$\begin{array}{l}\text { Modelo: } \\
\text { Área } \\
\end{array}$} & \multicolumn{2}{|c|}{$\begin{array}{c}\text { \%Diferencia Modelo: } \\
\text { Barra rectangular }\end{array}$} & \multicolumn{2}{|c|}{$\begin{array}{l}\text { \%Diferencia Modelo: } \\
\text { Barra irregular }\end{array}$} \\
\hline & & $\mathrm{Mx}$ & My & $\mathrm{Mx}$ & My & $\mathrm{Mx}$ & My \\
\hline G & 5 & 397128 & 426313 & $5,83 \%$ & $3,00 \%$ & $-8,18 \%$ & $-0,02 \%$ \\
\hline $\mathrm{H}$ & 10 & 1573720 & 1615884 & $1,14 \%$ & $1,89 \%$ & $1,03 \%$ & $-2,12 \%$ \\
\hline 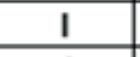 & 15 & 2631739 & 2719322 & $0,42 \%$ & $2,36 \%$ & $0,00 \%$ & $-2,00 \%$ \\
\hline $\mathrm{J}$ & 20 & 4047596 & 5228877 & $-3,27 \%$ & $-4,84 \%$ & $15,00 \%$ & $-1,06 \%$ \\
\hline $\mathrm{K}$ & 25 & 2231912 & 2711749 & $0,20 \%$ & $-1,39 \%$ & $28,76 \%$ & $24,62 \%$ \\
\hline $\mathbf{L}$ & 30 & 4616341 & 7876184 & $0,68 \%$ & $-0,40 \%$ & $20,88 \%$ & $13,38 \%$ \\
\hline \multicolumn{8}{|c|}{ Derivas } \\
\hline \multirow[t]{2}{*}{ Edificio } & \multirow{2}{*}{$\begin{array}{l}\text { Cantidad } \\
\text { de niveles }\end{array}$} & \multicolumn{2}{|c|}{$\begin{array}{l}\text { Modelo: } \\
\text { Área }\end{array}$} & \multicolumn{2}{|c|}{$\begin{array}{l}\text { \%Diferencia Modelo: } \\
\text { Barra rectangular }\end{array}$} & \multicolumn{2}{|c|}{$\begin{array}{l}\text { \%Diferencia Modelo: } \\
\text { Barra irregular }\end{array}$} \\
\hline & & $\mathrm{X}$ & $\mathrm{Y}$ & $\mathrm{x}$ & $\mathrm{Y}$ & $\mathrm{X}$ & $\mathrm{Y}$ \\
\hline $\mathbf{G}$ & 5 & 0,00053 & 0,00017 & $12,50 \%$ & $20,00 \%$ & $-25,00 \%$ & $-20,00 \%$ \\
\hline $\mathrm{H}$ & 10 & 0,00087 & 0,00027 & $3,85 \%$ & $0,50 \%$ & $-7,69 \%$ & $-12,50 \%$ \\
\hline 1 & 15 & 0,00080 & 0,00023 & $12,50 \%$ & $14,29 \%$ & $-33,33 \%$ & $-28,57 \%$ \\
\hline $\mathbf{J}$ & 20 & 0,00237 & 0,00073 & $4,23 \%$ & $0,34 \%$ & $-18,31 \%$ & $-18,18 \%$ \\
\hline $\mathbf{K}$ & 25 & 0,00337 & 0,00107 & $0,99 \%$ & $0,25 \%$ & $-19,80 \%$ & $-12,50 \%$ \\
\hline $\mathrm{L}$ & 30 & 0,00213 & 0,00063 & $1,56 \%$ & $5,26 \%$ & $-3,13 \%$ & $0,00 \%$ \\
\hline
\end{tabular}

Como puede apreciarse en los Tablas 4 y 5 , el modelado a través de elementos tipo barra con sección transversal rectangular presenta períodos mayores en las direcciones " $\mathrm{X}$ " $\mathrm{Y}$ " $\mathrm{Y}$ " en comparación con los obtenidos en modelos elaborados con elementos tipo área (a excepción del Edificio I en la dirección "Y"), por lo que se podría concluir que esta metodología conforma modelos más flexibles en dichas direcciones y por ende estos valores aumentan; en los casos más críticos se alcanzan diferencias de $+6,33 \%$ y $+6,54 \%$ para edificios ficticios y reales respectivamente. En contraparte, cuando se utilizan elementos tipo barra de sección transversal irregular, los períodos en las direcciones " $\mathrm{X}$ " $\mathrm{y}$ " $\mathrm{Y}$ " son menores a los obtenidos a través de modelos de elementos tipo área, es decir, caso contrario al de la técnica anterior, esta metodología constituye modelos más rígidos en tales direcciones, lo que repercute en la disminución de los períodos, siendo -25,41\% y $-22,48 \%$ las reducciones más significativas en cada caso. 
Los valores del Factor Espectral Dinámico (FED) utilizados para el cálculo del coeficiente sísmico de una estructura dependen directamente de los períodos principales asociados a esta. En general, este aspecto adquiere mayor relevancia a medida que se aumenta la cantidad de niveles de un edificio, ya que con la altura los períodos se desplazan hacia la derecha de la plataforma del espectro de diseño y en dicha zona conforme aumenta el período se reduce el valor del FED y viceversa, tal como se observa para el caso del Edificio K en la Figura 3, donde se aprecia la incidencia de la variación del período a través de la diferentes técnicas estudiadas en el FED utilizado para el cálculo de la fuerza sísmica.

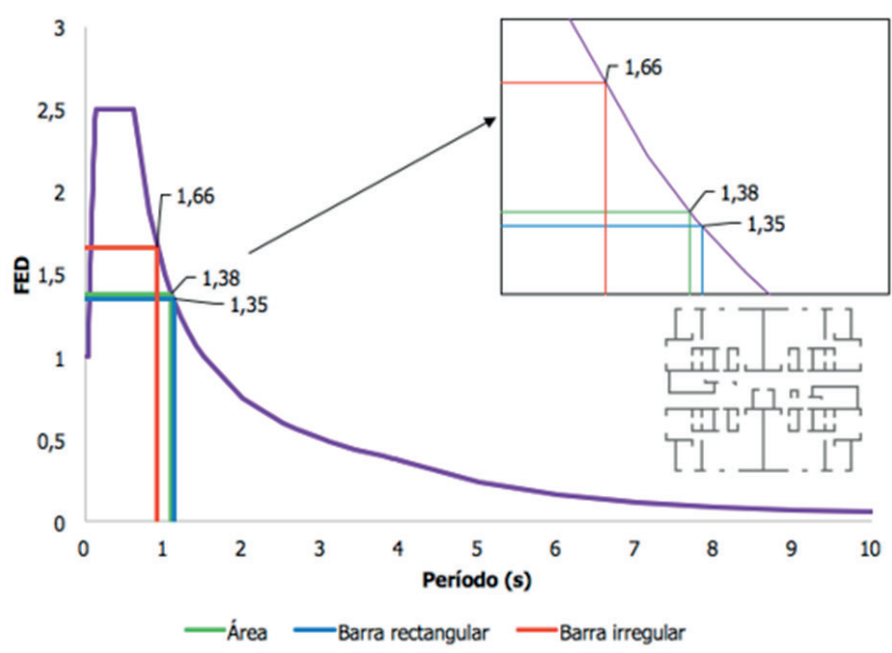

Figura 3. F para el Edificio K ( 25 niveles) en la dirección " $\mathrm{X}$ " a través de las diferentes técnicas

Además, los resultados muestran que los cortantes y momentos basales obtenidos a través de elementos tipo barra con sección transversal rectangular presentan diferencias porcentuales que para las magnitudes manejadas en el ámbito de la ingeniería civil y la incertidumbre del cálculo de la fuerza sísmica son aceptables. En el caso de las estructuras ficticias se obtienen diferencias máximas de $-1,41 \%$ y $-2,46 \%$ para el cortante y momento basal respectivamente, mientras que las estructuras reales presentan diferencias máximas de 6,59\% y 6,87\% para dichas fuerzas. Sin embargo, en el caso de los modelos construidos a través de elementos tipo barra de sección transversal irregular las diferencias porcentuales se incrementan sustancialmente, llegando a diferencias máximas de 18,00\% y 34,52\% en el cortante y momento basal de las estructuras ficticias, y presentando diferencias de $22,63 \%$ y $28,76 \%$ para el caso de edificios reales, por lo que si se toma como referencia el modelado a través de elemento tipo área se podría concluir que el uso de elementos tipo barra de sección transversal irregular para el modelado de edificios tipo muro, provoca que se sobreestime las fuerzas producidas durante la actividad sísmica, lo que repercute en mayores costos durante la etapa constructiva. Por otro lado, es importante mencionar que en esta investigación se toma como referencia los modelos de elementos tipo área dado que desde el punto de vista teórico son los que mejor se ajustan al comportamiento exhibido por los elementos chatos como los muros, sin embargo este en un aspecto que no se está comprobando, por lo que tampoco se puede asegurar si por el contrario el uso de elementos tipo área subestima las fuerzas sísmicas, con lo que se podría estar incumpliendo los factores de seguridad al deslizamiento y al vuelco

Finalmente, las diferencias porcentuales obtenidas en las derivas cuando se utilizan elementos tipo barra de sección transversal irregular son considerablemente mayores que cuando tales elementos se definen con secciones rectangulares. Es decir, nuevamente tomando como referencia el modelado a través de elementos tipo área, la metodología de modelado a través de elementos tipo barra de sección transversal irregular provoca que se subestime la flexibilidad de la estructura ante la acción del sismo. Por otro lado, aun cuando en el caso particular de los Edificios $\mathrm{K}$ e I modelados a través de elementos tipo barra de sección transversal regular estas diferencias son considerables, por lo general las razones de deriva de los edificios tipo muro no son el parámetro que rige el diseño de estas estructuras, por lo que los resultados anteriores no serían significativos en el diseño de dichos edificios a partir de los tipos de modelado estudiados en esta investigación.

La Figura 4 muestra el perfil de deformación del Edifico K en la dirección " $\mathrm{X}$ ", evidenciando que el modelado a través de elementos tipo barra con sección transversal irregular conforma modelos con mayor rigidez lateral.

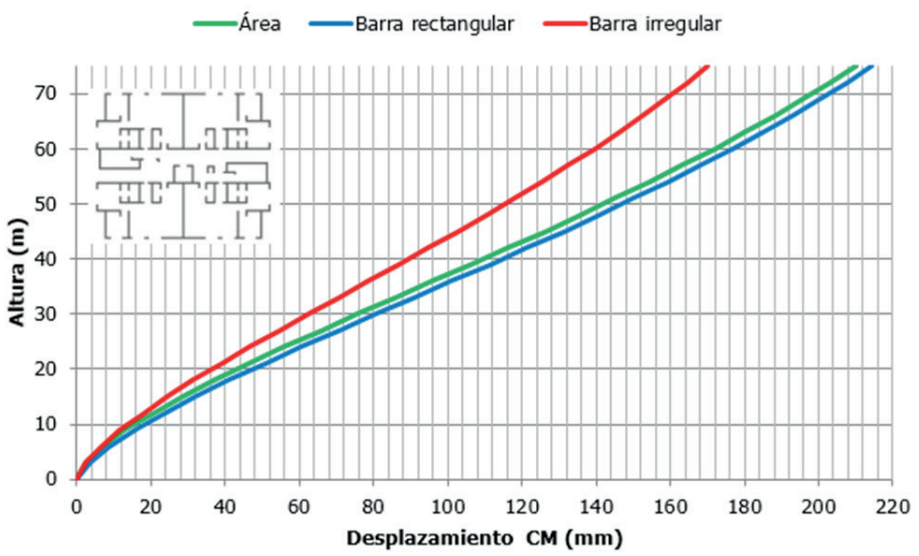

Figura 4. Deformada en "X" para Edificio K (25 niveles) con el sismo actuando en la misma dirección 


\section{CONCLUSIONES}

Los períodos obtenidos en las direcciones "X" $\mathrm{y}$ "Y" a través de los modelos de elementos tipo barra con sección transversal rectangular, alcanzan diferencias máximas de $+6,33 \%$ y $6,54 \%$ para edificios ficticios y reales respectivamente, en comparación con los valores arrojados por los modelos de elementos tipo área. Este es un indicativo de la rigidez, de modo que ambos modelos tienen una rigidez global lateral similar.

Por otro lado, en el caso de los modelos compuestos por elementos tipo barra con sección transversal irregular, se pueden llegar a obtener reducciones del período hasta en un $-25,41 \%$ y $-22,48 \%$ en las direcciones " $\mathrm{X}$ " $\mathrm{y}$ " $\mathrm{Y}$ " para edificios ficticios y reales en comparación con modelos de elementos tipo área, lo que implica que los modelos con elementos tipo barra con sección transversal irregular son más rígidos lateralmente.

Tomando como referencia la metodología de modelado con elementos tipo área, la estimación de períodos principales en las direcciones " $\mathrm{X}$ " $\mathrm{Y}$ " $\mathrm{Y}$ " al utilizar elementos tipo barra con sección transversal irregulares menos precisa que cuando se definen dichos elementos con secciones transversales rectangulares.

En términos de fuerzas cortantes y momentos en la base de la estructura, los modelos de elementos tipo barra con sección transversal rectangular presentan fuerzas sísmicas cuyos porcentajes de diferencia respecto a modelos elaborados con elementos tipo área son despreciables tomando en cuenta la incertidumbre manejada en el cálculo de la fuerza sísmica, por lo que para fines prácticos los valores de cortantes y momentos en la base obtenidos con estos modelos son comparables, llegando a obtener diferencias máximas de 6,59\% y 5,83\% para el cortante y momento basal respectivamente. En contraparte, los modelos de elementos tipo barra de sección transversal irregular presentan diferencias en las fuerzas de sismo considerablemente mayores que los elementos de tipo barra de sección transversal rectangular, 22,63\% y 34,52\% para el cortante y para el momento basal.

A nivel de desplazamientos la metodología de modelado a través de elementos tipo barra con sección transversal rectangular conforma modelos más flexibles que los de elementos tipo área, mientras que en el caso de los elementos tipo barra con sección transversal irregular los modelos adquieren mayor rigidez.
Las derivas obtenidas en modelos de elementos tipo barra con sección transversal rectangular son mayores que cuando se utilizan elementos de tipo área, caso contrario sucede cuando se utilizan elementos de tipo barra de sección transversal irregular.

En general, las respuestas estructurales globales que se pueden obtener a través de elementos de tipo barra con sección transversal rectangular se aproximan considerablemente a los resultados obtenidos a través de modelos de elementos tipo área, por lo que se podrían modelar edificios de tipo muro de hasta 30 niveles con ambas metodologías sin esperar diferencias significativas entre ambas técnicas.

\section{REFERENCIAS}

Ammar, A., \& Hassan, J. (2011). Shear wall analysis using framework method: comparison with shell element method and column analogy. Engineering and tech journal, 31, 3-13.

- Chandrupatla, T., \& Belegundu, A. (2011). Introducción al Elemento Finito en Ingeniería. Pennsylvania: Pearson.

- Chocano, A. (2003). Análisis estructural por el método matricial. Conceptos y aplicaciones. San José. Proyecto de graduación para optar por el grado de Licenciatura en Ingeniería Civil, Escuela de Ingeniería Civil, Universidad de Costa Rica.

- Colegio Federado de Ingenieros y Arquitectos. (Revisión 2014). Código Sísmico de Costa Rica 2010. San José: Editorial Tecnológica de Costa Rica.

Computers and Structures Inc. (2015). CSI Analysis reference manual. California.

Tan, M., \& Kubin, J. (2008). Comparison of practical approaches for modelling shearwalls in structural analyses of buildings. 14th World Conference on Earthquake Engineering, (págs. 1-8). Beijing. 